\title{
Jui-Lin Lee
}

\section{CLASSICAL MODEL EXISTENCE AND LEFT RESOLUTION}

\begin{abstract}
By analyzing what are necessary conditions in the proof [4] of the classical model existence theorem $C M E$ (every consistent set has a classical model), we present the left resolution Gentzen systems $\mathcal{R}(\neg,-)$, which prooftheoretically characterize $C M E$.

Keywords: classical propositional logic, classical model existence theorem, Gentzen system, resolution

1991 Mathematics Subject Classification: Primary: 03B05; Secondary: 03B20
\end{abstract}

\section{Introduction}

The extended completeness theorem of classical logic $(\Sigma \models \varphi$ implies $\Sigma \vdash \varphi$, for any $\Sigma, \varphi$ ), in most logic textbooks, is frequently proved by showing the following two statements:

CME: Every consistent set has a classical model (under the standard twovalued truth-functional semantics).

$R A A$ : If $\Sigma \nvdash \varphi$, then $\Sigma \cup\{\neg \varphi\}$ is consistent. ${ }^{1}$

The first statement, $C M E$, as the major step for proving completeness, may be weaker than the extended completeness theorem. It depends on what are

\footnotetext{
${ }^{1}$ In [4] the statement $C M E$ is named Classical Model Existence Theorem. This statement is also called Gödel-Malcev Theorem. Moreover, the statement RAA is also called Tarski's Lemma.
} 
viewed as logics. In [4] the author shows that, under the Hilbert-style proof procedure, in propositional logics the intuitionistic propositional logic IPL and some subintuitionistic or paraconsistent $\operatorname{logics}^{2}$ satisfy $C M E$. Hence in the propositional case naming $C M E$ the extended completeness theorem (or the strong completeness theorem) is not proof-theoretically appropriate, if what we consider are other propositional logics rather than just the classical propositional logic $C P L$.

However, the proof in [4] and other known completeness proofs give us only some sufficient conditions for CME (that is, the proof setting and the axiom schemes/inference rules of the given proof system form a set of conditions sufficient for proving $C M E$ ). One may naturally ask whether there is a weakest Hilbert system satisfying $C M E$ and/or what are the necessary and sufficient conditions for a logic satisfying $C M E$.

Consider the $\perp$-consistency case (here we take $\perp$ as a primitive symbol and the logic $\vdash$ as a binary relation over $2^{\mathcal{S}} \times \mathcal{S}$ with $\mathcal{S}$ the set of all sentences, and define that $\Sigma$ is $\perp$-consistent iff $(\Sigma, \perp) \notin \vdash)$. CME with respect to the $\perp$-consistency can be expressed as follows: For any $\Sigma \subseteq \mathcal{S}, \Sigma \models \perp$ implies $\Sigma \vdash \perp$. Since logics can be treated as relations over $2^{\mathcal{S}} \times \mathcal{S}$, , $\{(\Sigma, \perp) \mid \Sigma$ is not satisfiable $\} \subseteq L^{\prime}$ ' is a necessary and sufficient condition for any logic $L$ satisfying $C M E$ (with respect to the $\perp$-consistency). If we take the view that every $L \subseteq 2^{\mathcal{S}} \times \mathcal{S}$ is a logic, the weakest logic for $C M E$ (with respect to the $\perp$-consistency) is $\{(\Sigma, \perp) \mid \Sigma$ is not satisfiable $\}$. However, this semantic characterization of $C M E$ seems not useful from the proof-theoretical point of view. For example, it gives us no clue how to prove that the intuitionistic propositional logic IPL (or other weak logics) satisfies $C M E$.

Similarly, if we take the view that a logic is a consequence operation (see [10] for the definition), then the weakest consequence operation satisfying $C M E$ (with respect to the $\perp$-consistency) is

$$
C_{\perp}(\Sigma)= \begin{cases}\Sigma & \text { if } \Sigma \text { is satisfiable } \\ \Sigma \cup\{\perp\} & \text { else. }\end{cases}
$$

Again, due to the semantic nature in this description of $C_{\perp}$, this seems not helpful for showing that IPL (or other weak logics) satisfies $C M E$.

\footnotetext{
${ }^{2}$ This depends on which consistency one chooses. Though in the paraconsistent logic $\mathcal{H}\left(\mathrm{DT} 1, \mathrm{DT} 2, \mathrm{DA}_{\perp}^{*} ; M P\right)$ (in $[4, \mathrm{p} .192]$ ) the set $\{\perp\}$ is absolutely consistent (but not classically satisfiable and hence $C M E$ with respect to the absolute consistency does not hold in this system), $C M E$ with respect to the $\perp$-consistency holds in this system.
} 
This paper is organized as follows: In Section 2 we sketch the proof in [4] and analyze what are not necessary for proving $C M E$. In Section 3 we study the necessary conditions for proving $C M E$ from the abstract viewpoint that a logic is a Tarski-style relation (or a Gentzen-style relation). In Section 4 we design the left resolution Gentzen systems $\mathcal{R}(\neg,-)$ with two Gentzen rules (the left weakening with empty succedent rule $\left(1 \mathrm{~W}_{\Rightarrow \emptyset}\right)$ and the left resolution with empty succedent rule $\left.\left(\mathrm{l}_{\Rightarrow \emptyset}\right)\right)$ and initial sequents according to necessary conditions, and prove that $C M E$ holds for $\mathcal{R}(\neg,-)$. As a corollary, Gentzen's $L J$ also satisfies $C M E$ (see Corollary 4.16). We also prove that in general WRAA (see p. 340) or RAA does not imply the left resolution rule lRes. In Section 5 we discuss why the study of $C M E$ is useful.

\section{Background}

In this section we sketch the proof in [4], and then we analyze what conditions are not necessary in constructing a proof system satisfying $C M E$. Note that we will consider not only what axioms are needed, but also what kind of properties (in the Hilbert-style proof procedure) are necessary for proving $C M E$.

In [4], there are three steps to construct Hilbert systems satisfying $C M E$ :

(I) (Lindenbaum extension) Any consistent set $\Sigma$ can be enlarged to a maximal consistent set $\Delta$.

(II) (Negation completeness) The truth function of negation can be defined on $\Delta$ by adding some appropriate axiom schemes.

(III) (Truth functionality of connectives other than negation) The truth functions of other connectives can be defined on $\Delta$ by adding some appropriate axiom schemes.

We use the following settings as in [4].

Syntax of propositional logic. For simplicity we take one binary connective ' $\rightarrow$ ' (implication) and one unary connective ' $\neg$ ' (negation), that is, under $L(\rightarrow, \neg)$. We use a countably infinite set of atomic sentence symbols $P=$ $\left\{p_{0}, \ldots, p_{n}, \ldots\right\}$. Well-formed sentences are constructed inductively. We use meta symbols $\varphi, \psi, \ldots, A, B, C, \ldots$ for arbitrary sentences and $\Sigma, \Delta, \Pi$, ... for sets of (possibly infinitely many) sentences.

The classical semantics. All the semantic concepts like tautology, soundness, validity in this paper are classical, that is, by the standard two-valued truth functional semantics. $\models$ denotes the classical validity. 
The proof setting of Hilbert systems. $\mathcal{H}\left(A x_{1}, \ldots, A x_{m} ; R_{1}, \ldots, R_{n}\right)$ denotes the Hilbert system with axiom schemes $A x_{1}, \ldots, A x_{m}$ and inference rules $R_{1}, \ldots, R_{n}$, and $\mathcal{H}\left(A x_{1}, \ldots, A x_{m},-; R_{1}, \ldots, R_{n}\right)$ denotes all Hilbert systems which have inference rules $R_{1}, \ldots, R_{n}$ and contain at least axiom schemes $A x_{1}, \ldots, A x_{m}$. An axiom in Hilbert system $\mathcal{H}$ is an instance of certain axiom scheme in $\mathcal{H}$. A proof of $\psi$ from $\Sigma$ in Hilbert system $\mathcal{H}$ is a sequence $\varphi_{1}, \ldots$, $\varphi_{m}$ for some positive integer $m$ such that $\psi=\varphi_{m}$ and, for $1 \leqslant i \leqslant m$, each $\varphi_{i}$ is either from $\Sigma$, or an axiom of $\mathcal{H}$, or obtained by applying an inference rule of $\mathcal{H}$ to some of $\varphi_{1}, \ldots, \varphi_{i-1} . \Sigma \vdash_{\mathcal{H}} \psi$ means that there is a proof of $\psi$ from $\Sigma$ in $\mathcal{H}$.

We recall (see [8] and [4]) some general properties of Hilbert systems, which will be used for discussion later.

Proposition 2.1 (General proof properties for $\mathcal{H}$ ). For any sets of sentences $\Sigma, \Delta$ and for any sentence $\varphi$ :

(a) (Reflexivity) $\Sigma \vdash \varphi$ for any $\varphi \in \Sigma$.

(b) (Monotony) If $\Sigma \subseteq \Delta$ and $\Sigma \vdash \varphi$, then $\Delta \vdash \varphi$.

(c) (Transitivity) If $\Sigma \vdash \psi$ for each $\psi \in \Delta$, and $\Delta \vdash \varphi$, then $\Sigma \vdash \varphi$.

(d) (Finiteness) If $\Sigma \vdash \varphi$, then there exists a finite set $\Sigma_{0}$ such that $\Sigma_{0} \subseteq \Sigma$ and $\Sigma_{0} \vdash \varphi$.

(e) (Chain) If $\Delta_{0} \subseteq \ldots \subseteq \Delta_{i} \subseteq \ldots, \Delta=\bigcup_{i \in \mathbb{N}} \Delta_{i}$, and $\Delta \vdash \varphi$, then for some $n \in \mathbb{N}, \Delta_{n} \vdash \varphi$. (It can be derived by (b) and (d).)

(f) (Cut) If $\Sigma \vdash \varphi$ and $\Sigma \cup\{\varphi\} \vdash \psi$, then $\Sigma \vdash \psi$. (It can be derived by (a) and (c).)

Choosing the $\neg T$-consistency. There are several consistencies (simple consistency, absolute consistency, etc.). These consistencies are not always equivalent in weak proof systems. Therefore, we need to specify which consistency is taken for $C M E$. Here we choose the $\neg \top$-consistency (with $T$ being a fixed axiom in the Hilbert system we are going to construct) according to the following reasons:

- If the consistency is the unprovability of a fixed sentence, the maximal consistency of Lindenbaum extension (generated from a consistent set) can be proved without adding any axiom scheme.

- Taking the $\neg \top$-consistency with axiom $T$ is better than taking the $\perp$ consistency with $\neg \varphi=\varphi \rightarrow \perp$ (here $\perp$ is a primitive symbol). In the 
former case the definition of negation does not have to rely on implication. Therefore some proof-theoretically useful conditions about negation completeness will be directly highlighted in the former case, but not in the latter case.

Definition 2.2. (a) $\Sigma$ is $\neg \top$-consistent in $\mathcal{H}$ iff $\Sigma \nvdash_{\mathcal{H}} \neg \top$.

(b) $\Delta$ is maximal consistent (with respect to the consistency in concern) iff $\Delta$ is consistent and for any $\Pi$, if $\Delta \subseteq \Pi$ and $\Pi$ is consistent, then $\Delta=\Pi$.

(c) $\Sigma$ is negation complete iff for any sentence $\varphi$, exactly one of $\varphi, \neg \varphi$ is in $\Sigma$. ('Exactly one' means 'not both and at least one'.)

Here we choose $\top:={ }^{\prime}\left[p_{0} \rightarrow\left(p_{0} \rightarrow p_{0}\right)\right]$ ', which will be an axiom of the Hilbert system we are going to construct.

In the $\neg \top$-consistency case $C M E$ states that 'For any $\Sigma, \Sigma \nvdash \neg \top$ implies that $\Sigma$ has a classical model'. In the rest of this section the word 'consistency', if not specified, means the $\neg \top$-consistency. For $\mathcal{H}(-;-)$ we obtain

Lindenbaum's Lemma. If $\Sigma$ is consistent, then there is a maximal consistent extension of $\Sigma$.

Remark. The construction of maximal extension $\Delta$ in Lindenbaum's Lemma is done by enumerating all sentences $\varphi_{0}, \ldots, \varphi_{n}, \ldots$ and then defining a sequence of sets: $\Delta_{0}=\Sigma$ and for any $n \geqslant 0$

$$
\Delta_{n+1}= \begin{cases}\Delta_{n} \cup\left\{\varphi_{n}\right\} & \text { if } \Delta_{n} \cup\left\{\varphi_{n}\right\} \text { is consistent, } \\ \Delta_{n} & \text { else. }\end{cases}
$$

The consistency of $\Delta\left(=\bigcup_{n \in \mathbb{N}} \Delta_{n}\right)$ is proved by Proposition 2.1e. The maximality of $\Delta$ is proved by Proposition 2.1b. Note that Proposition 2.1a, Reflexivity, is not used in this proof.

Proposition 2.3 (Derivation closure property of $\Delta$ ). Let $\Delta$ be given as in the proof of Lindenbaum's Lemma with respect to $\mathcal{H}$. If $\Delta \vdash_{\mathcal{H}} \varphi$, then $\varphi \in \Delta$.

Remark. Proposition 2.3 is proved by Proposition 2.1f. Actually, any maximal consistent set $\Delta$ satisfies the derivation closure property because the only Lindenbaum extension of $\Delta$ is $\Delta$ itself. 
We will take the following two axiom schemes

$$
\begin{aligned}
& A \rightarrow(B \rightarrow A) \\
& {[A \rightarrow(B \rightarrow C)] \rightarrow[(A \rightarrow B) \rightarrow(A \rightarrow C)]}
\end{aligned}
$$

into $\mathcal{H}$, so that the well-known deduction theorem ( $D T$ for short) holds in $\mathcal{H}$. (With this, $\top:=$ ' $p_{0} \rightarrow\left(p_{0} \rightarrow p_{0}\right)$ ' becomes an axiom of $\mathcal{H}$.)

Theorem 2.4 (Deduction theorem for $\mathcal{H}(\mathrm{DT} 1, \mathrm{DT} 2,-; M P)$ ). Let $\Sigma$ be a set of sentences and $\varphi$ and $\psi$ be any sentences. Then $\Sigma \cup\{\varphi\} \vdash \psi$ implies $\Sigma \vdash \varphi \rightarrow \psi$.

Now we show that $\Delta$ is negation complete by adding new axiom schemes. Three possibly applicable axiom schemes are $\left(\mathrm{DA}_{0}\right)$ (a special case of Duns Scotus' law), $\left(\mathrm{E}_{\neg}\right.$ ) (the law of elimination of $\neg$ with respect to the $\neg \top$ consistency), and $\left(\mathrm{I}_{\neg}\right.$ ) (the law of introduction of $\neg$ with respect to the $\neg$ T-consistency) $:^{3}$

$$
\begin{aligned}
& \neg A \rightarrow(A \rightarrow \neg \top) \\
& (\neg A \rightarrow \neg \top) \rightarrow A \\
& (A \rightarrow \neg \top) \rightarrow \neg A
\end{aligned}
$$

Theorem 2.5 (Negation completeness of $\Delta$ ). For Hilbert systems $\mathcal{H}(\mathrm{DT} 1$, $\left.\mathrm{DT} 2, \mathrm{DA}_{0}, \mathrm{E}_{\neg},-; M P\right)$ and $\mathcal{H}\left(\mathrm{DT} 1, \mathrm{DT} 2, \mathrm{DA}_{0}, \mathrm{I}_{\neg},-; M P\right)$, the set $\Delta$ in the proof of Lindenbaum's Lemma is negation complete.

Proof. (Recall the proof in [4] for analysis.) Not-Both: To avoid that $\Delta$ contains both $\varphi$ and $\neg \varphi$ but still can not prove $\neg \top$, we add $\left(\mathrm{DA}_{0}\right)$. If $\varphi, \neg \varphi \in$ $\Delta$, by $\left(\mathrm{DA}_{0}\right)$ (replacing $A$ by $\varphi$ ) and $M P$ twice, $\Delta \vdash \neg \top$, a contradiction. At-Least-One: If both $\varphi$ and $\neg \varphi$ are not in $\Delta$, then $\Delta \cup\{\varphi\} \vdash \neg \top$ and $\Delta \cup\{\neg \varphi\} \vdash \neg T$. Take schemes (DT1) and (DT2) so that DT holds. By $D T, \Delta \vdash(\varphi \rightarrow \neg \top)$ and $\Delta \vdash(\neg \varphi \rightarrow \neg \top)$. To show that $\Delta \vdash \neg \top$ we may add just one of $\left(\mathrm{E}_{\neg}\right),\left(\mathrm{I}_{\neg}\right)$. By any of $\left(\mathrm{E}_{\neg}\right),\left(\mathrm{I}_{\neg}\right)$ and $M P$ twice, $\Delta \vdash \neg \top$, a contradiction. Note that $\left(\mathrm{I}_{\neg}\right)$ is provable in $I P L$, but $\left(\mathrm{E}_{\neg}\right)$ is not.

Finally for the truth functionality of ' $\rightarrow$ ' on $\Delta$, except taking $M P$ and axiom (DT1), we add one more axiom (DA) (Duns Scotus' law-the law of denial of the antecedent):

$$
\neg A \rightarrow(A \rightarrow B)
$$

\footnotetext{
${ }^{3}$ Of course, $\left(\mathrm{I}_{\neg}\right)$ follows from (DT1), (DT2), $\left(\mathrm{DA}_{0}\right)$ and $\left(\mathrm{E}_{\neg}\right)$, thus in theorems $2.5,2.6$ and 2.7 one can leave only cases for $\mathcal{H}\left(\ldots, I_{\neg}, \ldots\right)$.
} 
TheOrem 2.6 (Truth functionality of ' $\rightarrow$ ' on $\Delta$ ). For both Hilbert systems $\mathcal{H}\left(\mathrm{DT} 1, \mathrm{DT} 2, \mathrm{DA}, \mathrm{I}_{\neg},-; M P\right)$ and $\mathcal{H}\left(\mathrm{DT} 1, \mathrm{DT} 2, \mathrm{DA}, \mathrm{E}_{\neg},-; M P\right)$, the set $\Delta$ in the proof of Lindenbaum's Lemma satisfies the following property for any $\varphi$ and $\psi$ :

$$
\varphi \rightarrow \psi \in \Delta \text { iff } \varphi \notin \Delta \text { or } \psi \in \Delta .
$$

Proof. (Recall the proof in [4] for analysis.) Here $\left(\mathrm{DA}_{0}\right)$ is replaced by the more general (DA) and then $\Delta$ is negation complete by Theorem 2.5.

$(\Rightarrow)$ Let $\varphi \rightarrow \psi \in \Delta$. If $\varphi \in \Delta$, by $M P \Delta \vdash \psi$. By Proposition 2.3, $\psi \in \Delta$.

$(\Leftarrow)$ If $\psi \in \Delta$, then by (DT1) and $M P, \Delta \vdash \varphi \rightarrow \psi$ and then by Proposition 2.3, $\varphi \rightarrow \psi \in \Delta$. If $\varphi \notin \Delta$, by the negation completeness of $\Delta, \neg \varphi \in \Delta$. By $(\mathrm{DA}), \vdash \neg \varphi \rightarrow(\varphi \rightarrow \psi)$. By $M P, \Delta \vdash \varphi \rightarrow \psi$. Вy Proposition 2.3, $\varphi \rightarrow \psi \in \Delta$.

Theorem 2.7 ( $C M E$ with respect to the $\neg T$ consistency). For both systems $\mathcal{H}\left(\mathrm{DT} 1, \mathrm{DT} 2, \mathrm{DA}, \mathrm{I}_{\neg},-; M P\right)$ and $\mathcal{H}\left(\mathrm{DT} 1, \mathrm{DT} 2, \mathrm{DA}, \mathrm{E}_{\neg},-; M P\right)$, every consistent set has a classical model. ${ }^{4}$

Proof. (Recall the proof in [4] for analysis.) By Lindenbaum's Lemma, any consistent set $\Sigma$ can be extended to a maximal consistent set $\Delta$. Take (DT1), (DT2) so that DT holds. By Theorem 2.5 (since $\left(\mathrm{DA}_{0}\right)$ is a special case of (DA)), such $\Delta$ is negation complete. By Theorem 2.6, the truth function of ' $\rightarrow$ ' on $\Delta$ is defined. Now one can define an interpretation $\mu: P \rightarrow\{T, F\}$ : for any atomic sentence $p_{i}, \mu\left(p_{i}\right)=T$ iff $p_{i} \in \Delta$. It is proved by induction that (according to the truth functions of ' $\rightarrow$ ' and ' $\neg$ ') the interpretation $\bar{\mu}$ extended from $\mu$ has the following property: for any sentence $A, \bar{\mu}(A)=T$ iff $A \in \Delta$. Then $\mu$ is a classical model of $\Sigma \subseteq \Delta$.

Now we discuss what are not necessary in constructing a proof system satisfying $C M E$.

I. On what are not necessary for proving Lindenbaum's Lemma. (This part has been briefly discussed in [4], p. 184.) As we have seen, in Hilbert systems we need not add any axiom scheme for proving Lindenbaum's Lemma (with respect to the $\neg T$-consistency). If we are looking for any system which precisely characterizes CME under any proof procedure, in above proof what conditions are not necessary for proving Lindenbaum's Lemma are about the Hilbert-style proof procedure.

\footnotetext{
${ }^{4}$ Note that $\mathcal{H}\left(\mathrm{DT} 1, \mathrm{DT} 2, \mathrm{DA}, \mathrm{I}_{\neg} ; M P\right)$ is the $\{\neg, \rightarrow\}$-fragment of $I P L$.
} 
Reflexivity is not necessary for Lindenbaum's Lemma. This has been observed in [1]. In [1] the Lindenbaum-Asser theorem, which is an abstract generalization of Lindenbaum's Lemma, states that in any finite monotonic logic $L$ (i.e., $L$ satisfies Proposition 2.1bd) and for any sentence $\tau$ and any set $\Sigma$ such that $\Sigma \nvdash_{L} \tau$ (i.e., $\Sigma$ is $\tau$-consistent), a relatively maximal extension of $\Sigma$ (i.e., a maximal $\tau$-consistent Lindenbaum extension of $\Sigma$ ) exists.

One may argue that Proposition 2.3 (the derivation closure property of $\Delta$ ) is used in above proof and to prove Proposition 2.3 one may need Cut (i.e., Proposition 2.1f), which is a consequence of Transitivity and Reflexivity. Then it seems that we need a finite normal logic $L$ (i.e., $L$ satisfies Proposition 2.1abcd) for proving Lindenbaum's Lemma and Proposition 2.3.

We do not have to take a finite normal logic. Firstly, as we will see, Proposition 2.3 is not necessary: We will choose other sufficient conditions for negation completeness and truth functionality (for connectives other than ' $\neg$ ') on $\Delta$ so that there is no need to use Proposition 2.3. Secondly, even if one uses Proposition 2.3, taking Proposition 2.1ef will suffice. Although Proposition 2.1f is derived from Transitivity and Reflexivity, this derivation is conducted under the Hilbert-style proof procedure. There are, under different proof procedures, some proof systems in which Reflexivity fails and Proposition 2.1ef hold. (For example, $\{(\Sigma, \perp) \mid \Sigma$ is not satisfiable $\}$.)

II. On what are not necessary for proving the negation completeness of $\Delta$. For Not-Both in the proof of Theorem 2.5, the inference ' $\varphi$ and $\neg \varphi$ infer $\neg \top^{\prime}$ (denoted $\left.\{\varphi, \neg \varphi\} \Rightarrow \neg \top\right)$ will suffice. Then the axiom scheme $\left(\mathrm{DA}_{0}\right)$ and the rule $M P$ need not be used here.

For At-Least-One in the proof of Theorem 2.5, we may avoid using DT, $M P$ and take one of the following conditions instead of $\left(\mathrm{I}_{\neg}\right)$ and $\left(\mathrm{E}_{\neg}\right)$.

WRAA: If $\Sigma \cup\{\varphi\} \vdash \neg \top$, then $\Sigma \vdash \neg \varphi$.

$R A A: \quad$ If $\Sigma \cup\{\neg \varphi\} \vdash \neg \top$, then $\Sigma \vdash \varphi$.

However, none of these two conditions is necessary. The following condition is a necessary condition for At-Least-One.

lRes: If $\Sigma \cup\{\varphi\} \vdash \neg \top$ and $\Sigma \cup\{\neg \varphi\} \vdash \neg \top$, then $\Sigma \vdash \neg \top$.

Note that WRAA, RAA and lRes can be easily formulated as inference rules with discharged assumptions in natural deduction systems or inference rules in Gentzen systems. It seems not possible to formulate any of them as inference rules without discharged assumptions in Hilbert systems, and this 
is why in Hilbert systems we employ $D T$ for discharging assumptions at the meta level.

III. On what are not necessary in proving truth functionality of ' $\rightarrow$ ' on $\Delta$. Consider the truth functionality condition of ' $\rightarrow$ ' on $\Delta$, ' $\varphi \rightarrow \psi \in \Delta$ iff $\varphi \notin \Delta$ or $\psi \in \Delta$ '. What are necessary are the following conditions (for any sentences $A, B):\{A, \neg B, A \rightarrow B\} \Rightarrow \neg \top,\{B, \neg(A \rightarrow B)\} \Rightarrow \neg \top$, $\{\neg A, \neg(A \rightarrow B)\} \Rightarrow \neg \top$. (This is inspired by $\{(\Sigma, \perp) \mid \Sigma$ is not satisfiable $\}$, in which $\perp$ is the only possible succedent.)

Alternatively, from the equivalent statement ' $\varphi \rightarrow \psi \in \Delta$ iff ether $\varphi \notin \Delta$ and $\psi \notin \Delta$, or $\varphi \notin \Delta$ and $\psi \in \Delta$, or $\varphi \in \Delta$ and $\psi \in \Delta$ ' we get the following necessary conditions: $\{A, \neg B, A \rightarrow B\} \Rightarrow \neg \top,\{A, B, \neg(A \rightarrow B)\} \Rightarrow \neg \top$, $\{\neg A, B, \neg(A \rightarrow B)\} \Rightarrow \neg \top,\{\neg A, \neg B, \neg(A \rightarrow B)\} \Rightarrow \neg \top$.

Now $M P, D T$ or Proposition 2.3 are not necessary conditions. To see this we shall prove that all necessary conditions mentioned above are sufficient for proving CME (see Section 4).

Remark. The main difference of [1] and [4] is that in [1] in order to prove the completeness theorem the semantics taken is the semantics of all relatively maximal theories (of a finite normal logic). In our terminology, it means that for any sentence $\tau$ and any set $\Sigma$ such that $\Sigma \nvdash \tau$ all Lindenbaum extensions of $\Sigma$ with respect to $\tau$-consistency are put into the semantics. In contrast in [4] we consider a weaker statement $C M E$ and deal with relatively maximal extensions with respect to a fixed sentence $\neg \top$.

Remark. Under the Hilbert-style proof procedure it is not possible to get a weakest Hilbert system satisfying $C M E$. Consider the $\perp$-consistency case. If $\{(\Sigma, \perp) \mid \Sigma$ is not satisfiable $\}$ can be expressed as a Hilbert system, it is clear that such Hilbert system has no axiom (because the only possible case $(\emptyset, \perp)$ is not valid). Hence above construction (by adding axiom schemes) is probably not a good approach to construct the weakest Hilbert system (if there is any) satisfying $C M E$.

\section{On the necessary conditions for $C M E$}

In this section we discuss necessary conditions for proving $C M E$. Here we treat any (Tarski-style) proof system $L$ as a collection of arguments $(\Sigma, \varphi)$ with single-sentence succedent. In this way $L$ is seen as a relation $L \subseteq 2^{\mathcal{S}} \times \mathcal{S}$ (where $\mathcal{S}$ is the set of all sentences). We use $\Sigma \vdash_{L} \varphi$ to denote $(\Sigma, \varphi) \in L$. When $L$ is fixed, we write $\vdash$ instead of $\vdash_{L} . C P L=\{(\Sigma, \varphi) \mid \Sigma \models \varphi\}$, that is, $\vdash_{C P L}$ is the classical validity $\models$. Note that $\models$ satisfies Proposition 2.1. 
A logic $L$ is sound iff $L \subseteq C P L$. In this case we will introduce a primitive symbol $\perp$ which will be always interpreted false and we say that $\Sigma$ is $\perp$ consistent in $L$ iff $(\Sigma, \perp) \notin L$.

As mentioned in Section 1, ' $\{(\Sigma, \perp) \mid \Sigma$ is not satisfiable $\} \subseteq L$ ' is a necessary and sufficient condition for $L$ satisfying $C M E$ (with respect to the $\perp$-consistency). Since this characterization is not proof-theoretically useful, we extract the following proof-theoretically useful conditions from the proof sketch in Section 2 and show that they are necessary in this section.

Recall that $C M E$ for $L$ with respect to the $\perp$-consistency states that 'For any $\Sigma, \Sigma \models \perp$ implies $\Sigma \vdash_{L} \perp$ '.

Theorem 3.8 (Finiteness and monotony with respect to $\Sigma \vdash \perp$ ). If $L$ satisfies $C M E$ (w.r.t. $\perp$-consistency) and $L$ is sound, then

(a) $\Sigma \vdash_{L} \perp$ implies that there is a finite set $\Sigma^{\prime}$ such that $\Sigma^{\prime} \subseteq \Sigma$ and $\Sigma^{\prime} \vdash_{L} \perp$.

(b) $\Sigma \vdash_{L} \perp$ and $\Sigma \subseteq \Delta$ imply $\Delta \vdash_{L} \perp$.

Proof. By the soundness of $L$, then by the finiteness/monotony of $\models$, and then by the $C M E$ of $L$.

Theorem 3.9 (Not-Both for negation completeness and truth functionality of ' $\rightarrow$ '). If $L$ satisfies $C M E$, then the following conditions hold for any sentence $A, B:\{A, \neg A\} \vdash_{L} \perp,\{A, \neg B, A \rightarrow B\} \vdash_{L} \perp,\{\neg A, \neg(A \rightarrow B)\} \vdash_{L} \perp$, $\{B, \neg(A \rightarrow B)\} \vdash_{L} \perp,\{\neg \top\} \vdash_{L} \perp,\{\perp\} \vdash_{L} \perp$. (Here ' $T$ ' and ' $\perp$ ' are 0-ary connectives and $\mu(T)=T$ and $\mu(\perp)=F$, for any truth assignment $\mu$, and they are in consideration with respect to systems using them as primitive symbols.)

Proof. All of these are valid arguments with the conclusion $\perp$. Since $L$ satisfies $C M E$ (w.r.t. $\perp$-consistency), all of them are provable in $L$.

Next we look for the necessary condition for At-Least-One.

Theorem 3.10 (At-Least-One for negation completeness). If $L$ satisfies $C M E$ (w.r.t. $\perp$-consistency) and $L$ is sound, then:

$$
\Sigma \cup\{\varphi\} \vdash_{L} \perp \text { and } \Sigma \cup\{\neg \varphi\} \vdash_{L} \perp \text { imply } \Sigma \vdash_{L} \perp \text {. }
$$

Proof. Assume that $\Sigma \cup\{\varphi\} \vdash_{L} \perp$ and $\Sigma \cup\{\neg \varphi\} \vdash_{L} \perp$. Since $L$ is sound, $\Sigma \cup\{\varphi\} \models \perp$ and $\Sigma \cup\{\neg \varphi\} \models \perp$. For any truth assignment $v$ such that $v \models \Sigma$, either $v \models \varphi$ or $v \models \neg \varphi$. From any of these cases we derive $v \models \perp$. Hence $\Sigma \models \perp$. Since $L$ satisfies $C M E, \Sigma \vdash_{L} \perp$. 
The next two properties, $N E C$ (the negated extended completeness) and $E C$ (the extended completeness), are not necessary conditions for $C M E$.

$N E C$ : For any $\Sigma$ and $\varphi, \Sigma \models \neg \varphi$ implies $\Sigma \vdash_{L} \neg \varphi$.

$E C$ : For any $\Sigma$ and $\varphi, \Sigma \models \varphi$ implies $\Sigma \vdash_{L} \varphi$.

Anyway, these two proof-theoretically useful conditions relate to WRAA (for any $\Sigma$ and $\varphi$, if $\Sigma \cup\{\varphi\} \vdash_{L} \perp$, then $\Sigma \vdash_{L} \neg \varphi$ ) and $R A A$ (for any $\Sigma$ and $\varphi$, if $\Sigma \cup\{\neg \varphi\} \vdash_{L} \perp$, then $\Sigma \vdash_{L} \varphi$ ) respectively. Note that $C P L$ (or equivalently $\models)$ satisfies WRAA and $R A A$.

Theorem 3.11. (a) If $L$ is sound and satisfies $N E C$, then $L$ satisfies WRAA.

(b) If $L$ satisfies $C M E$ and WRAA, then $L$ satisfies NEC.

(c) If $L$ is sound and satisfies $E C$, then $L=C P L$ and $L$ satisfies $R A A$.

(d) If $L$ satisfies $C M E$ and $R A A$, then $L$ satisfies $E C$ (i.e., $C P L \subseteq L$ ).

Proof. (a) Suppose that $\Sigma \cup\{\varphi\} \vdash_{L} \perp$. Since $L$ is sound, $\Sigma \cup\{\varphi\} \models \perp$. According to $W R A A$ for $\models, \Sigma \models \neg \varphi$. Since L satisfies $N E C, \Sigma \vdash_{L} \neg \varphi$.

(b) Suppose that $\Sigma \models \neg \varphi$. According to $\models, \Sigma \cup\{\varphi\} \models \perp$. Since $L$ satisfies $C M E, \Sigma \cup\{\varphi\} \vdash_{L} \perp$. By WRAA of $L, \Sigma \vdash_{L} \neg \varphi$.

(c) and (d) are trivial.

Remark. We may also consider logics as Gentzen-style relations, i.e., any (Gentzen-style) proof system as a relation $G \subseteq 2^{\mathcal{S}} \times 2^{\mathcal{S}}$. (The Tarski-style relation $L \subseteq 2^{\mathcal{S}} \times \mathcal{S}$ can be converted into $G_{L}=\left\{(\Sigma,\{\varphi\}) \mid \Sigma \vdash_{L} \varphi\right\}$.) The Gentzen-style classical semantics $\Vdash_{C}$ is defined as follows: $\Sigma \Vdash_{C} \Delta$ iff for every truth assignment $v$, either $v$ interprets $\varphi$ false for some $\varphi \in \Sigma$ or $v$ interprets $\psi$ true for some $\psi \in \Delta$. For a Gentzen-style relation $G$, we use $\Sigma \Vdash_{G} \Delta$ to denote $(\Sigma, \Delta) \in G$. When $G$ is fixed, we write $\Vdash$ instead of $\Vdash_{G}$. We then consider $\bigvee \emptyset$ consistency, i.e., $\Sigma$ is consistent in $G$ iff $\Sigma \nVdash_{G} \emptyset$. Then the properties $N E C, E C, W R A A, R A A, C M E$, lRes can be translated under this setting. (e.g., $G$ is sound iff $G$ is a subset of $\vdash_{C}$; $G$ satisfies $C M E$ iff $\Sigma \Vdash_{C} \emptyset$ implies $\Sigma \Vdash_{G} \emptyset$, for any $\Sigma \subseteq \mathcal{S}$.) Then theorems 3.8, 3.9, 3.10 and 3.11 can reformulated and proved under this setting.

Note that $\Vdash_{C}$ has the following properties.

1. If $\Sigma \cap \Pi \neq \emptyset$ then $\Sigma \Vdash_{C} \Pi$.

2. If $\Sigma \subseteq \Sigma^{\prime}, \Pi \subseteq \Pi^{\prime}$ and $\Sigma \Vdash_{C} \Pi$, then $\Sigma^{\prime} \Vdash_{C} \Pi^{\prime}$.

3. If $\Sigma \Vdash_{C} \Pi$, then there exist finite sets $\Sigma_{0}$ and $\Pi_{0}$ such that $\Sigma_{0} \subseteq \Sigma$, $\Pi_{0} \subseteq \Pi$, and $\Sigma_{0} \Vdash_{C} \Pi_{0}$. (By 2 we also have $\Sigma_{0} \Vdash_{C} \Pi$ and $\Sigma \Vdash_{C} \Pi_{0}$.) 
4. If $\Delta_{0} \subseteq \ldots \subseteq \Delta_{i} \subseteq \ldots, \Delta=\bigcup_{i \in \mathbb{N}} \Delta_{i}$, and $\Delta \Vdash_{C} \Pi$, then for some $n \in \mathbb{N}$, $\Delta_{n} \Vdash_{C} \Pi$.

5. If $\Sigma \cup\{\varphi\} \Vdash_{C} \emptyset$ and $\Sigma \cup\{\neg \varphi\} \Vdash_{C} \emptyset$, then $\Sigma \Vdash_{C} \emptyset$.

\section{Left resolution Gentzen systems}

In this section we construct Gentzen systems which exactly characterize $C M E$. We use $L(\neg,-)$, that is, we take one unary connective ' $\neg$ ' (negation) and none or any other connectives as we like. ${ }^{5}$

We use $\Gamma, \Delta, \Sigma, \ldots$ to denote sets of sentences. These sets are possibly infinite or empty. A sequent $S$ is of the form $\Gamma \Rightarrow \Delta$, where sets of sentences $\Gamma, \Delta$ are the antecedent and the succedent of $S$, respectively. Here we choose $\Gamma, \Delta$ being sets instead of multisets or sequences so that we need not introduce the structural rules of Exchange and Contraction, which are not necessary conditions (in proof procedure) for $C M E$. When $\Delta$ is the empty set $\emptyset$, we use $\Gamma \Rightarrow$ to denote $\Gamma \Rightarrow \emptyset$. Since the meaning of $\Gamma \Rightarrow \Delta$ in sequent calculus is that $\bigwedge \Gamma \rightarrow \bigvee \Delta$ is a classical tautology, we choose the $\bigvee \emptyset$-consistency (and then we need not introduce $\perp$ nor $\neg \top$ ).

As an example we consider the following system $\mathcal{R}(\neg, \supset, \equiv)$, with two binary connectives ' $\supset$ ' (implication), ' $\equiv$ ' (equivalence).

Definition 4.12. The left resolution Gentzen system $\mathcal{R}(\neg, \supset, \equiv)$ is a Gentzen system with initial sequents for any sentences $A$ and $B$ :

$$
\begin{aligned}
& A, \neg A \Rightarrow \\
& A, B, \neg(A \supset B) \Rightarrow \\
& \neg A, B, \neg(A \supset B) \Rightarrow \\
& \neg A, \neg B, \neg(A \supset B) \Rightarrow \\
& A, \neg B, A \supset B \Rightarrow \\
& A, B, \neg(A \equiv B) \Rightarrow \\
& \neg A, B, A \equiv B \Rightarrow \\
& \neg A, \neg B, \neg(A \equiv B) \Rightarrow \\
& A, \neg B, A \equiv B \Rightarrow
\end{aligned}
$$

and, for any sets $\Gamma$ and $\Pi$ of sentences (they may be infinite), two Gentzen rules: left weakening with empty succedent $\left(1 \mathrm{~W}_{\Rightarrow \emptyset}\right)$, and left resolution with

\footnotetext{
${ }^{5}$ Warning: ' $\perp$ ' is not necessarily a primitive symbol and we do not take the convention that $\neg \varphi=\varphi \supset \perp$.
} 
empty succedent $\left(\mathrm{lR}_{\Rightarrow \emptyset}\right)$.

$$
\begin{aligned}
& \frac{\Gamma \Rightarrow}{\Pi \cup \Gamma \Rightarrow} \\
& \frac{\Gamma \cup\{A\} \Rightarrow \quad \Gamma \cup\{\neg A\} \Rightarrow}{\Gamma \Rightarrow}
\end{aligned}
$$

A proof of a sequent $\Gamma \Rightarrow \Delta$ in $\mathcal{R}(\neg, \supset, \equiv)$ is a finite proof tree with a single root $\Gamma \Rightarrow \Delta$ and initial sequents of $\mathcal{R}(\neg, \supset, \equiv)$ labeled at the top nodes, and each node-label connected with the label of the immediate successor node-label (if any) according to one of the rules of $\mathcal{R}(\neg, \supset, \equiv$ ). $\mathcal{R}(\neg, \supset, \equiv) \vdash \Gamma \Rightarrow \Delta$ denotes that there is a proof of the sequent $\Gamma \Rightarrow \Delta$ in $\mathcal{R}(\neg, \supset, \equiv)$.

Similarly, we use $\mathcal{R}\urcorner^{\top}(\neg, \supset, \equiv)$ to denote the left resolution Gentzen system with modified Gentzen rules $\left(\mathrm{lW}_{\Rightarrow \neg T}\right),\left(\mathrm{lR}_{\Rightarrow \neg T}\right)$ and modified initial sequents by adding $\neg \top$ to succedents of all sequents.

Remark. The Gentzen rule $\left(1 \mathrm{~W}_{\Rightarrow \emptyset}\right)$ is a necessary condition by Theorem $3.8 \mathrm{~b}$, with the modification described at the end of Section 4. The initial sequent (1), inspired from Not-Both of negation completeness, is a necessary condition by modified Theorem 3.9. The initial sequents (2)-(5), which offer the truth functionality of ' $\supset$ ' on $\Delta$, are necessary conditions by modified Theorem 3.9. We call the initial sequents (2)-(5) the truth functional sequents for ' $\supset$ '. Similarly one can prove that the initial sequents (6)-(9), the truth functional sequents for ' $\equiv$ ', are necessary conditions for any logic which has ' $\neg$ ', '三' and satisfies CME.

In general we can construct the left resolution Gentzen system $\mathcal{R}(\neg,-)$ which has the rules $\left(1 \mathrm{~W}_{\Rightarrow \emptyset}\right)$ and $\left(\mathrm{l}_{\Rightarrow \emptyset}\right)$, the initial sequent $(1)$, and all the truth functional sequents (as initial sequents) for the connectives we choose. These left resolution Gentzen systems are negation based.

Remark (Does the rule $\left(\mathrm{l}_{\Rightarrow \emptyset}\right)$ deserve the name 'resolution'?). The left resolution rule $\left(\mathrm{IR}_{\Rightarrow \emptyset}\right)$ is a necessary condition, by Theorem 3.10. Usually the well-known resolution method applies to $S=\bigwedge_{i \in I} C_{i}$ (where $I$ is not necessarily finite and each clause $C_{i}$ is a finite set of literals). If there is a derivation of $\emptyset$ by the standard resolution rule

$$
\frac{\Sigma \cup\{p\} \quad \Gamma \cup\{\neg p\}}{\Sigma \cup \Gamma}
$$

from finitely many $C_{i}$ of $S$, then $S$ is unsatisfiable. This can be seen as applying right resolution rule on sequents $\Rightarrow C_{i}(i \in I)$. In this case, $\Rightarrow C_{i}$ from $S$ behave like assumptions of a proof in Hilbert systems. 
What we do in $\mathcal{R}(\neg,-)$ is different. Every $\mathcal{R}(\neg,-)$ has a fixed set of initial sequents (with respect to the corresponding connectives) from which all unsatisfiable sets of sentences can be derived: By the compactness theorem every unsatisfiable set $\Sigma$ has a finite subset $\Sigma^{\prime}$ which is unsatisfiable. In $\mathcal{R}(\neg,-)$ from its initial sequents we can derive $\Sigma^{\prime} \Rightarrow$ first, and then by $\left(\mathrm{lW}_{\Rightarrow \emptyset}\right.$ ) we derive $\Sigma \Rightarrow$. In this case, all initial sequents of $\mathcal{R}(\neg,-)$ behave like axioms in Hilbert systems.

There is a natural connection between our systems $\mathcal{R}(\neg,-)$ and the method of extension, introduced by [9]. Tsejtin's method of extension, as described in $[2$, p. 20], is done as follows: For a given sentence $A$, we generate a set of clauses $\Gamma_{A}$ so that $A$ is falsifiable iff there exists an interpretation which satisfies every clause of $\Gamma_{A}$. Then $A$ is a tautology iff $\Gamma_{A}$ is unsatisfiable iff there is a resolution refutation from $\Gamma_{A}$. The construction of $\Gamma_{A}$ is done by firstly introducing propositional variable $x_{B}$ for every $B$ which is a subformula of $A$, then identifying $\overline{x_{\neg B}}$ with $x_{B}$ (also $x_{\neg B}$ with $\overline{x_{B}}$ ), and then putting into $\Gamma_{A}$ the clause $\left\{\overline{x_{A}}\right\}$ and other clauses constructed according to the truth functionality. We explain the case $\wedge$ as an example: If $B \wedge C$ is a subformula of $A$, then we add to $\Gamma_{A}$ the following clauses $\left\{\overline{x_{B \wedge C}}, x_{B}\right\}$, $\left\{\overline{x_{B \wedge C}}, x_{C}\right\},\left\{x_{B \wedge C}, \overline{x_{B}}, \overline{x_{C}}\right\}$. Other connectives can be done in a similar way.

Our left resolution Gentzen system is a dual of Tsejtin's method. If we put the clauses of Tsejtin's method on the right of $\Rightarrow$, convert $\overline{x_{B}}$ to $\neg B$ and $x_{B}$ to $B$, use the $\neg \Rightarrow$ rule in Gentzen's sequent calculus to convert everything to the left of $\Rightarrow$, and remove ' $\neg \neg$ ', if necessary, we will get one set of truth functional sequents for ' $\wedge$ ': $B \wedge C, \neg B \Rightarrow ; B \wedge C, \neg C \Rightarrow ; \neg(B \wedge C), B, C \Rightarrow$. (This set of initial sequents is provably equivalent to $B \wedge C, \neg B, C \Rightarrow ; B \wedge$ $C, \neg B, \neg C \Rightarrow ; B \wedge C, B, \neg C \Rightarrow ; \neg(B \wedge C), B, C \Rightarrow$ by $\left(\mathrm{lW}_{\Rightarrow \emptyset}\right)$ and $\left(\mathrm{lR}_{\Rightarrow \emptyset}\right)$.) From our view Tsejtin's method is the right resolution method.

The following theorem can be easily proved by induction for any $\mathcal{R}(\neg,-)$. Theorem 4.13. Let $\mathcal{R}$ be a left resolution Gentzen system $\mathcal{R}(\neg,-)$.

(a) (Soundness) If $\mathcal{R} \vdash \Gamma \Rightarrow \Delta$, then $\Gamma \models \Delta$.

(b) (Finiteness) If $\mathcal{R} \vdash \Gamma \Rightarrow \Delta$, then there is a finite set $\Gamma_{0}$ such that $\Gamma_{0} \subseteq \Gamma$ and $\mathcal{R} \vdash \Gamma_{0} \Rightarrow \Delta$.

(c) If $\mathcal{R} \vdash \Gamma \Rightarrow \Delta$, then $\Delta=\emptyset$ (i.e., $\Delta$ is the empty set).

Now we prove the classical model existence theorem for any left resolution Genzten system $\mathcal{R}$. For this we take the $\bigvee \emptyset$-consistency: $\Gamma$ is $\bigvee \emptyset$-consistent in $\mathcal{R}$ iff $\mathcal{R} \nvdash \Gamma \Rightarrow$. 
Theorem 4.14 (Classical model existence theorem for $\mathcal{R}(\neg,-)$ ). If $\Gamma$ is $\vee \emptyset$-consistent in $\mathcal{R}$, then $\Gamma$ has a classical model.

Proof. Enumerate all sentences $\varphi_{0}, \ldots, \varphi_{n}, \ldots$ and define the following sequence of sets of sentences:

$$
\begin{aligned}
\Delta_{0} & =\Gamma, \\
\vdots & \\
\Delta_{n+1} & = \begin{cases}\Delta_{n} \cup\left\{\varphi_{n}\right\} & \text { if } \mathcal{R} \nvdash \Delta_{n} \cup\left\{\varphi_{n}\right\} \Rightarrow, \\
\Delta_{n} & \text { else. }\end{cases}
\end{aligned}
$$

Then by mathematical induction $\Delta_{n}$ is consistent for any $n \in \mathbb{N}$.

Let $\Delta:=\bigcup_{i \in \mathbb{N}} \Delta_{i}$. Then $\mathcal{R} \nvdash \Delta \Rightarrow$, i.e. $\Delta$ is consistent in $\mathcal{R}$. (Indeed, if not, by Theorem 4.13b there is a finite set $\Gamma_{0} \subseteq \Delta$ such that $\mathcal{R} \vdash \Gamma_{0} \Rightarrow$. Since $\Delta=\bigcup_{i \in \mathbb{N}} \Delta_{i}$, there is an $n \in \mathbb{N}$ such that $\Gamma_{0} \subseteq \Delta_{n}$ and by $\left(1 \mathrm{~W}_{\Rightarrow \emptyset}\right)$ we get $\mathcal{R} \vdash \Delta_{n} \Rightarrow$, a contradiction.)

$\Delta$ is maximal. Indeed, for any $n \in \mathbb{N}$, if $\varphi_{n} \notin \Delta$, then $\mathcal{R} \vdash \Delta_{n} \cup\left\{\varphi_{n}\right\} \Rightarrow$. By $\left(\mathrm{lW}_{\Rightarrow \emptyset}\right), \mathcal{R} \vdash \Delta \cup\left\{\varphi_{n}\right\} \Rightarrow$. Then any proper extension of $\Delta$ is not consistent.

$\Delta$ is negation complete. Not-Both: if $\varphi, \neg \varphi \in \Delta$, then $\mathcal{R} \vdash \varphi, \neg \varphi \Rightarrow$, by (1); so we obtain $\mathcal{R} \vdash \Delta \Rightarrow$, by $\left(\mathrm{lW}_{\Rightarrow \emptyset}\right)$, a contradiction. At-Least-One: if $\varphi \notin \Delta$ and $\neg \varphi \notin \Delta$, then by the definition of $\Delta$ and $\left(\mathrm{lW}_{\Rightarrow \emptyset}\right), \mathcal{R} \vdash \Delta \cup\{\varphi\} \Rightarrow$ and $\mathcal{R} \vdash \Delta \cup\{\neg \varphi\} \Rightarrow$. Thus, by $\left(\mathrm{lR}_{\Rightarrow \emptyset}\right), \mathcal{R} \vdash \Delta \Rightarrow$, a contradiction.

For the truth-functionality of other connectives on $\Delta$, it is proved by the negation completeness and the truth functional sequents for the corresponding connectives. For example, the initial sequent $A, \neg B, A \supset B \Rightarrow$, the negation completeness of $\Delta$ and the rule $\left(1 W_{\Rightarrow \emptyset}\right)$ force that: if $A \in \Delta$ and $B \notin \Delta$, then $(A \supset B) \notin \Delta$.

Now we prove that all truth-functional sequents in $\mathcal{R}(\neg,-)$ are independent.

Theorem 4.15. In $\mathcal{R}(\neg, \supset, \equiv)$, the initial sequents (2)-(9) are independent.

Proof. We take the initial sequent $(2)$ in $\mathcal{R}(\neg, \supset, \equiv)$ as an example. Assume that the initial sequent $(2)$ can be derived in $[\mathcal{R}(\neg, \supset, \equiv)-(2)]$, for any $A, B$, i.e.,

$$
[\mathcal{R}(\neg, \supset, \equiv)-(2)] \vdash A, B, \neg(A \supset B) \Rightarrow
$$


We add one more initial sequent

$$
A, B, A \supset B \Rightarrow
$$

By above assumption, adding $(*)$ to the system we can still prove (2):

$$
[\mathcal{R}(\neg, \supset, \equiv)-(2)+(*)] \vdash A, B, \neg(A \supset B) \Rightarrow
$$

and $(*)$ can be proved because it is an initial sequent:

$$
[\mathcal{R}(\neg, \supset, \equiv)-(2)+(*)] \vdash A, B, A \supset B \Rightarrow
$$

Thus, by $\left(\mathrm{lR}_{\Rightarrow \emptyset}\right)$, we obtain

$$
[\mathcal{R}(\neg, \supset, \equiv)-(2)+(*)] \vdash A, B \Rightarrow
$$

Now $[\mathcal{R}(\neg, \supset, \equiv)-(2)+(*)]$ is sound, if we interpret ' $\equiv$ ' the usual way and ' $A \supset B$ ' by ' $\neg A$ ' in $[\mathcal{R}(\neg, \supset, \equiv)-(2)+(*)]$. But then by Theorem 4.13a, $\{A, B\} \models \bigvee \emptyset$, for any $A, B$. Then $[\mathcal{R}(\neg, \supset, \equiv)-(2)+(*)]$ is not sound under this interpretation, a contradiction.

Note that sometimes the initial sequent $(1)$ in $\mathcal{R}(\neg,-)$ may be redundant: $A, \neg A \Rightarrow$ can be derived from the truth functional sequents for ' $\supset$ ' (or ' $\equiv$ ') by $\left(\mathrm{lR}_{\Rightarrow \emptyset}\right)$.

Remark. The unsigned classical propositional tableau method offers a way of constructing a left resolution proof. Assume that $\Gamma$ is not satisfiable. Then by the compactness theorem there is a finite set $\Gamma_{0}$ such that $\Gamma_{0} \subseteq \Gamma$ and $\Gamma_{0}$ is not satisfiable. Then $\Gamma \Rightarrow$ is derived by applying $\left(\mathrm{WW}_{\Rightarrow \emptyset}\right)$ to $\Gamma_{0} \Rightarrow$. As an example, let us assume that $\Gamma_{0} \Rightarrow$ is $\Pi,(B \equiv C) \Rightarrow$. If we apply unsigned 三-rule in tableau method on $B \equiv C$, this will generate to four nodes: Two discarded nodes $\Pi, B, \neg C,(B \equiv C) \Rightarrow$ and $\Pi, \neg B, C,(B \equiv C) \Rightarrow$ (which can be derived by $\left(\mathrm{lW}_{\Rightarrow \emptyset}\right)$ from two truth functional sequents $B, \neg C,(B \equiv$ $C) \Rightarrow$ and $\neg B, C,(B \equiv C) \Rightarrow)$, and two other nodes $\Pi, B, C,(B \equiv C) \Rightarrow$ and $\Pi, \neg B, \neg C,(B \equiv C) \Rightarrow$. These four nodes are merged by applying $\left(\mathrm{R}_{\Rightarrow \emptyset}\right)$ three times. Repeatedly apply tableau rules on every non-discarded node. If the finite set $\Gamma_{0}$ is unsatisfiable, then each path either reaches a discarded node, or eventually reaches a node (a sequent) which contains $A$, $\neg A$ in its antecedent for some $A$ (and this can be derived from $A, \neg A \Rightarrow$ by $\left.\left(1 \mathrm{~W}_{\Rightarrow \emptyset}\right)\right)$.

Now we apply this to the intuitionistic propositional logic in the form of Gentzen's $L J$. 
Corollary 4.16. The classical model existence theorem holds in $L J$, that is, if $\Gamma \models \bigvee \emptyset$, then there is a finite set $\Gamma_{0} \subseteq \Gamma$ such that $\Gamma_{0} \Rightarrow$ is provable in $L J{ }^{6}$

Proof. Consider the left resolution Gentzen system $\mathcal{R}(\neg, \wedge, \vee, \supset)$. One can easily verify that all initial sequents of $\mathcal{R}(\neg, \wedge, \vee, \supset)$ (by modifying sets into sequents) are provable in $L J$. Also the finite version of the two rules $\left(1 \mathrm{~W}_{\Rightarrow \emptyset}\right)$ and $\left(\mathrm{lR}_{\Rightarrow \emptyset}\right)$ (that is, with all the antecedents being finite sets) can be easily derived in $L J$. (What we prove here actually show that all initial sequents and (finite version of) rules of $\mathcal{R}(\neg, \wedge, \vee, \supset$ ) are admissible in $L J$.)

If $\Gamma \models \bigvee \emptyset(=\perp)$, then by the compactness theorem there is a finite subset $\Gamma_{0} \subseteq \Gamma$ such that $\Gamma_{0} \models \bigvee \emptyset$. By Theorem 4.14, $\Gamma_{0} \Rightarrow$ is provable in $\mathcal{R}(\neg, \wedge, \vee, \supset)$. Therefore, $\Gamma_{0} \Rightarrow$ is provable in $L J$.

Note that $\mathcal{R}(\neg,-)+R A A$ (in sequent form) is a Gentzen system for $C P L$.

At the end of this section we prove that in general WRAA or $R A A$ does not imply the left resolution rule lRes. For simplicity we consider $L(\neg, \supset)$.

THEOREM 4.17. There are Gentzen systems which contain all initial sequents of the left resolution Gentzen system $\mathcal{R}(\neg, \supset)$, satisfy $\left(\mathrm{lW}_{\Rightarrow \emptyset}\right)$ and WRAA (or RAA), but in which CME does not hold.

Proof. The idea is that we will take the generalization of left weakening rule for any sets $\Gamma, \Pi$ and $\Delta$ (which may be infinite)

$$
\frac{\Gamma \Rightarrow \Delta}{\Pi \cup \Gamma \Rightarrow \Delta}
$$

and all initial sequents of $\mathcal{R}(\neg, \supset)$ into the Gentzen system $G$, and then add other rules and initial sequents so that $G$ has WRAA admissible.

If we apply $W R A A$ (on sentence $C$ ) to $\Sigma, C \Rightarrow$ with $\Sigma$ containing one of the antecedents of the initial sequents (1)-(5), we can simply add the following rule to $G$ :

$$
\frac{\Gamma \Rightarrow}{\Gamma \Rightarrow \neg C}
$$$$
\left(\mathrm{rW}_{\Rightarrow \emptyset+\neg}\right)
$$

In this case we can derive $\Sigma \Rightarrow$ by $\left(\mathrm{lW}_{\Rightarrow}\right)$ (from one of the initial sequents (1) $-(5))$ and then derive $\Sigma \Rightarrow \neg C$ by $\left(\mathrm{rW}_{\Rightarrow \emptyset+\neg}\right)$ in $G$. On the other hand, if $\Sigma$ does not contain any of the antecedents of the initial sequents (1)-(5),

\footnotetext{
${ }^{6}$ Here $\models$ is the classical validity.
} 
$C$ must be introduced from one of the initial sequents (1)-(5) (that is, not introduced by $\left.\left(\mathrm{lW}_{\Rightarrow}\right)\right)$. Then we may derive this by "applying WRAA" to the corresponding initial sequent and then by $\left(\mathrm{lW}_{\Rightarrow}\right)$. (Note that once WRAA is applied, we cannot apply $\left(\mathrm{rW}_{\Rightarrow \emptyset+\neg}\right)$ to any resulting sequent $\Gamma \Rightarrow \neg C$, which has a non-empty succedent.) Instead of taking $W R A A$ as a rule, we add the following new sequents into $G$ so that $W R A A$ is admissible with respect to the initial sequents (1)-(5) (and any sequent obtained from these by $(\mathrm{lW} \Rightarrow))$ :

$$
\begin{aligned}
& \neg A \Rightarrow \neg A \\
& A \Rightarrow \neg \neg A \\
& A, B \Rightarrow \neg \neg(A \supset B) \\
& A, \neg(A \supset B) \Rightarrow \neg B \\
& B, \neg(A \supset B) \Rightarrow \neg A \\
& \neg A, B \Rightarrow \neg \neg(A \supset B) \\
& \neg A, \neg(A \supset B) \Rightarrow \neg B \\
& B, \neg(A \supset B) \Rightarrow \neg \neg A \\
& \neg A, \neg B \Rightarrow \neg \neg(A \supset B) \\
& \neg A, \neg(A \supset B) \Rightarrow \neg \neg B \\
& \neg B, \neg(A \supset B) \Rightarrow \neg \neg A \\
& A, \neg B \Rightarrow \neg(A \supset B) \\
& A, A \supset B \Rightarrow \neg \neg B \\
& \neg B, A \supset B \Rightarrow \neg A
\end{aligned}
$$

Then the Gentzen system $G$ with initial sequents (1)-(5), (10)-(23) and inference rules $\left(\mathrm{lW}_{\Rightarrow}\right),\left(\mathrm{rW}_{\Rightarrow \emptyset+\neg}\right)$ has $W R A A$ admissible. However, $G$ does not satisfy $C M E$ : $A, \neg B, \neg \neg(A \supset B) \Rightarrow$ cannot be derived in $G$. If it were derivable, it must be derived by $\left(\mathrm{lW}_{\Rightarrow}\right)$ from one of the initial sequents. This is clearly not possible.

The case of $R A A$ is similar. We take rules $\left(\mathrm{IW}_{\Rightarrow}\right)$ and

$$
\frac{\Gamma \Rightarrow}{\Gamma \Rightarrow C}
$$

and then we take initial sequents (1)-(5) and add some more initial sequents so that $R A A$ becomes admissible. For initial sequent $A, \neg A \Rightarrow$, as an example, we add $A \Rightarrow A$ and $\neg \neg C \Rightarrow C$ (this is the resulting sequent by applying $R A A$ on $\neg C$ when $A$ is $\neg C$ ). Since $R A A$ can be applied only on sequents 
with empty succedent, we generate finitely many new sequents from initial sequents (1)-(5) by $R A A$. Adding these new sequents to the Gentzen system we make $R A A$ admissible. Again, $A, \neg B, \neg \neg(A \supset B) \Rightarrow$ cannot be derived in this Gentzen system by the same reason.

Note that the cases for $\mathcal{R}^{\top}(\neg,-)$ or $\mathcal{R}^{\perp}(\neg,-)$ can be proved in a similar way.

\section{Concluding remarks}

We should explain why the study of $C M E$ is useful. Firstly, according to [5], the study of $C M E$ is related to the general theory of consistencies. Intuitively, a set $\Sigma$ is inconsistent iff $\Sigma$ has something wrong, and (probably) most people will agree that at least $\Sigma$ should not have a classical model. Define that $C U=\{\Sigma \mid \Sigma$ is not classically satisfiable $\}$ and consider any nonempty collection $\boldsymbol{\Phi} \subseteq C U$. Then for any Tarski-style relation $L \subseteq 2^{\mathcal{S}} \times \mathcal{S}$ we can define $\boldsymbol{\Phi}$-consistency as follows: a set $\Sigma$ is $\boldsymbol{\Phi}$-consistent in $L$ iff every element of $\boldsymbol{\Phi}$ is not a subset of $\left\{\varphi \mid \Sigma \vdash_{L} \varphi\right\}$. It is clear that $C U$-consistency implies $\boldsymbol{\Phi}$ consistency for any nonempty $\boldsymbol{\Phi}$. Then $C M E$ with respect to $\boldsymbol{\Phi}$-consistency for a sound $L$ implies that $C U$-consistency in $L$ and $\boldsymbol{\Phi}$-consistency in $L$ are equivalent. Examples of consistencies are: $\boldsymbol{\Phi}=\{\{\perp\}\}$ (the $\perp$-consistency), $\boldsymbol{\Phi}=\{\{\varphi, \neg \varphi\} \mid \varphi \in \mathcal{S}\}$ (the simple consistency), $\boldsymbol{\Phi}=\{\mathcal{S}\}$ (the absolute consistency). For any sound $L$, the $C U$-consistency is the strongest one and the absolute consistency is the weakest one (any $\boldsymbol{\Phi}$-consistency implies the absolute consistency since $\mathcal{S}$ is maximal in $C U$ ). When $C M E$ with respect to the absolute consistency holds in sound $L$, it means that all consistencies in $L$ are equivalent.

Secondly, the proof-theoretical analysis of the exact conditions for $C M E$ gives us not only a proof-theoretical characterization of $C M E$ (in terms of the left resolution Gentzen systems, see Section 4), but also a way to seek for weak logics satisfying $C M E$, which is applicable to paraconsistent logics, subintuitionistic logics (see [4]), substructural logics, modal logics (weak extensions of $B C I W$ logic, see [7]), predicate logics (see [6]). Note that Glivenko's Theorem holds in some of these weak logics (see $[6,7]$ ). Note that $C M E$ does not hold in minimal logic: $\{A \rightarrow \perp,(A \rightarrow B) \rightarrow \perp\}$ is $\perp$ consistent in minimal logic (using Segerberg's axiomatization, see [3, p. 307]) but it does not have a classical model.

Finally, we propose some research problems:

1. What algebraic structures/properties correspond to these results in $[4,6$, $7]$ ? Is there any new syntactic translation based on these? 
2. What is the relationship between models/semantics of logics satisfying the same model existence property? One may consider other model existence property, e.g., that of intuitionistic predicate logic, which is not equivalent to $C M E$.

\section{References}

[1] Béziau. J.-Y., "Sequents and bivaluations", Logique et Analyse 176 (2001), $373-94$.

[2] Buss, S. R., "An introduction to proof theory", pp. 1-78 in Handbook of Proof Theory, Stud. Logic Found. Math., 137, North-Holland, Amsterdam, 1998.

[3] Epstein, R. L., The Semantic Foundations of Logic. Vol. 1: Propositional logics, 2nd edn., Oxford University Press, 1995.

[4] Lee, J.-L., "Classical model existence theorem in propositional logics", pp. 179197 in Perspectives on Universal Logic, J-Y. Béziau and A. Costa-Leite (eds.), Polimetrica, Monza, Italy, 2007.

[5] Lee, J.-L., "Consistency and satisfiability", presented in Taiwan Philosophical Association 2007 Annual meeting, on October 21, 2007.

[6] Lee, J.-L., "Classical model existence theorem in subclassical predicate logics. I", accepted and to appear in Trends in Logic: Towards Mathematical Philosophy, D. Makinson, J. Malinowski and H. Wansing (eds.), in the book series "Trends in Logic" - Studia Logica Library, 2008.

[7] Lee, J.-L., "Glivenko's Theorem for weak normal modal logics", submitted to Advances in Modal Logic 2008, 2008.

[8] Tarski, A., "Fundamentale Begriffe der Methodologie der deduktiven Wissenschaften. I.", Monatshefte für Mathematik und Physik 37 (1930), 361-404.

[9] Tsejtin, G.S., "On the complexity of derivation in propositional logic", Studies in Constructive Mathematics and Mathematical Logic 2 (1968), 115-125.

[10] Wójcicki, R., Theory of Logical Calculi, Synthese Library, vol. 199, Dordrecht: Kluwer Academic Publishers Group Press, 1988.

Jui-Lin LEE

Department of Philosophy

National Chung-Cheng University

Taiwan

jlleeLOGICIAN@gmail.com 